\title{
双能量 CT 血管造影在自发性颅内出血中的诊断价值
}

\section{Diagnostic Value of Dual Energy CT Angiography in Spontaneous Intracranial Hemorrhage 郑倩倩 张恒 王荵}

\author{
Qianqian Zheng Heng Zhang Rui Wang
}

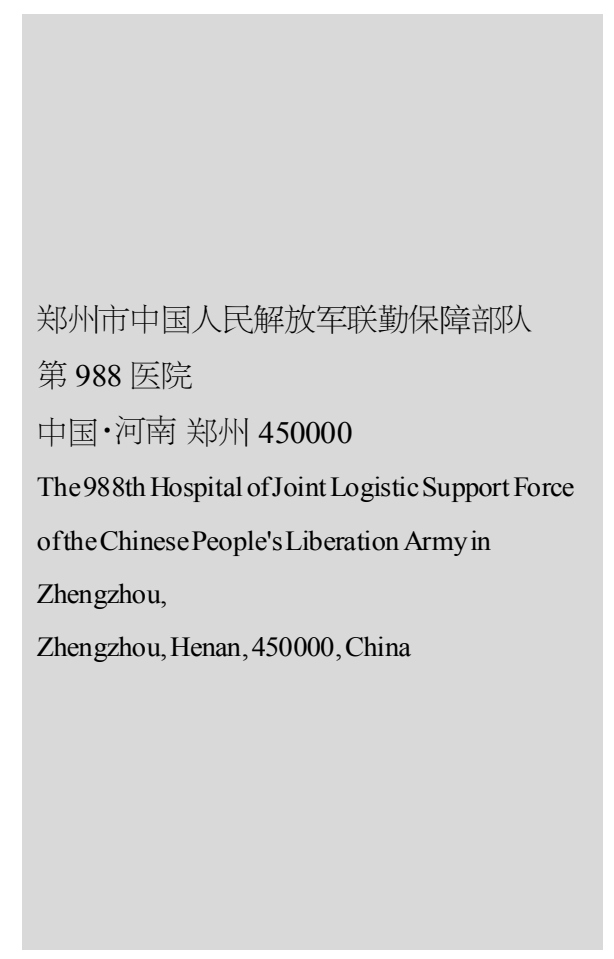

【摘要】在临床诊断过程中, 应用高科技技术对提高诊断效率, 保障人民身体健康具有积 极意义。论文以自发性颖内出血疾病患者为研究对象, 通过常规血管造影术和双能量 CT 血 管造影术检测效果的对比, 发现应用双能量 CT 血管造影术的患者的平均检测时间及平均 辐射剂量都远小于使用常规血管造影术的患者, 得出了双能量 CT 血管造影术具有检测时 间短、伤害小、准确率高的优势, 值得在临床诊断中大力推广及应用的结论, 以期为其他医 院工作人员提供参考和借鉴。

【Abstract】In the process of clinical diagnosis, the application of high-tech technology is of positive significance to improve the efficiency of diagnosis and ensure people's health. Based on spontaneous intracranial hemorrhage disease patients as the research object, through conventional angiography and dual energy CT angiography effect contrast, found that the application of dual energy CT angiography in patients with average detection time and the average radiation dose is much smaller than the conventional angiography in patients, it is concluded that the dual energy CT angiography has short detection time, small damage, the advantage of high accuracy, is worth promoting and application in clinical diagnosis conclusion, so as to provide reference for other hospital workers.

【关键词】双能量 CT 血管造影; 自发性杪内出血; 诊断价值

【Keywords】dual-energy CT angiography; spontaneous intracranial hemorrhage; diagnostic value 【DOI 】10.36012/pmr.v1i3.927

\section{1 引言}

自发性颅内出血,即非外部伤害造成, 而是由于各种自发 性因素导致脑内动脉、静脉及毛细血管破裂而引起的颉内出 血现象,其属于多因素疾病,会受到遗传因素和环境因素的共 同作用。造成自发性颉内出血的原因主要是高血压,其他的还 有先天性血管瘤、凝血障碍、淀粉样变性血管病、动静脉畸形 等原因。同时, 自发性颅内出血作为常见脑部血管疾病, 具有 发病急、病情重、死残概率高等特点, 受到了社会和医院的广 泛关注。早期发现治疗能够实现准确沴断出血原因, 可帮助医 师了解患者情况, 采取有效治疗措施, 能够挽救患者的生命健 康。本文随机选取了 2018 年 8 月一 2019 年 6 月我院收治的 58 例自发性颅内出血患者, 在患者知情并同意研究治疗的前 提下, 将患者平均分为观察组和对照组, 观察组采用双能量 CT 血管造影方法,对照组采用常规 CT 血管造影,每组 29 例, 对比并分析检测结果, 获得如下数据和结论。

\section{2 资料与方法}

\section{1 一般资料}

本文选取我院 2018 年 8 月一 2019 年 6 月收治的 58 例自 发性颅内出血患者, 并在尊重患者及家属意愿的前提下, 将患 者平均分为了观察组和对照组。其中, 观察组患者共 29 例, 男 性为 17 例, 女性为 12 例, 年龄范围为 20 77 岁, 平均年龄为 (58.2 \pm 6.3$)$ 岁; 对照组患者共 29 例, 男性患者 15 例, 女性患者 14 例, 年龄分布在 $(56 \pm 7.2)$ 岁。每组患者及其家属都被告知本 次研究具体内容及可能存在的风险,并自愿签署知情同意书。 通过将两组患者的一般资料进行对比, 可知差异 $P>0.05$, 并不 具备统计学意义, 因此可以继续展开研究。

\section{2 检测方法}

对于对照组患者来说, 通过常规 CT 血管造影进行检测, 选择美国 GE 公司生产的 16 排螺旋 CT 仪器, 对肘前静脉进 行穿刺, 并利用压力注射器, 在检查前 1 小时注人 $100 \mathrm{~mL}$ 浓度 
为 $20 \mathrm{mg} / \mathrm{mL}$ 的非离子型造影剂, 注入速率控制在 $3 \mathrm{~mL} / \mathrm{s}$, 并在 完成注射后等待约 20s 后进行扫描。其中, 16 排螺旋 CT 仪器 的参数设置如下: 设置螺距为 $110 \mathrm{~mm}$, 层厚为 $113 \mathrm{~mm}$, 管电压 设置在 120 140kV, 电流控制在 300 432 $\mathrm{mA}$ 。对于观察组患者 来说, 采用双能量 CT 血管造影检测, 选用德国西门子公司的 双源 CT 仪器, 应先对定位图像进行扫描, 随后进行双能量扫 描, 在扫描过程中要求患者成平卧题为, 并从足静脉注射浓度 为 $350 \mathrm{mg} / \mathrm{mL}$ 的碘海醇 $75 ~ 90 \mathrm{~mL}$, 随后注射 $50 \mathrm{~mL}$ 生理盐水, 并设置团注跟踪智能触发扫描模式, 将主动脉设置为靶血管。 双源 $\mathrm{CT}$ 仪器的参数设置如下：设置准直器宽度为 $128 \mathrm{~mm} \times$ $0.6 \mathrm{~mm}$, 球管电压分别设置为 $80 \mathrm{kV} 、 140 \mathrm{kV}$, 矩阵设置为 $512 \times$ 512 , 螺距 $0.7 \mathrm{~mm}$, 球管旋速 $0.5 \mathrm{~s} / \mathrm{r}$, 触发嗞值设定为 $100 \mathrm{HU}^{[1]}$ 。

\section{3 评定标准}

血管造影影像的诊断是由放射科资深医师负责, 并由两 名及两名以上的医师获得一致的诊断结果, 方可确定为最终 诊断结果, 并将手术结果及数字减影血管造影术 (DSA) 检查 标准,对两种检测方法的效果进行对比分析。

\section{4 统计学分析}

本文应用 SPSS18.0 统计学软件对上述研究获得的数据进 行统计, 用 表示计量资料, 用 $t$ 检验结果, 并用 $\chi^{2}$ 检验计数资 料, 以例数 $(\%)$ 来表示数据对比差异, 若 $P<0.05$, 则表明差异 具有统计学意义。

\section{3 结果}

通过对比对照组和观察组两组患者辐射剂量和扫描时间, 可发现对照组患者平均辐射剂量几乎比观察组患者平均辐射 剂量高一倍; 观察组患者的平均扫描时间远小于对照组患者平 均扫描时间, 且差异 $P<0.05$, 具有统计学意义,如表 1 所示。

表 1 对照组和观察组患者辐射剂量、扫描时间对比

\begin{tabular}{c|c|c|c}
\hline 组别 & 例数 & 平均辐射剂量 $(\mathrm{mGy})$ & 平均扫描时间 $(\mathrm{s})$ \\
\hline 观察组 & 29 & $328.59 \pm 28.76$ & $75.41 \pm 6.74$ \\
\hline 对照组 & 29 & $734.81 \pm 36.42$ & $98.27 \pm 12.48$ \\
\hline$t$ & & 5.712 & 11.257 \\
\hline$P$ & & $<0.05$ & $<0.05$ \\
\hline
\end{tabular}

另外, 通过对比观察组和对照组检测结果的准确率, 可知 观察组患者存在 2 例误诊现象,检验准确率为 $93.10 \%$, 对照 组患者存在 3 例误诊现象, 检验准确率为 $89.7 \%$, 对比两组之 间的差异为 $P>0.05$, 不具备统计学意义 ${ }^{[2]}$

\section{4 讨论}

由于自发性颉内出血疾病发病快、病情严重,致残致死率 相比其他疾病要高, 因此需要对发病原因进行及时确认, 并采
取对症措施予以治疗, 方可有效提高患者恢复健康的概率, 并 降低患者的伤残概率。在临床诊断过程中, CT 血管造影 (CTA) 数字减影血管造影术 (DSA) 都是自发性颉内出血疾病 的常用检验方法,数字减影血管造影术 (DSA) 是当前诊断该 疾病的基本标准,但由于检查过程中具有创伤性,并且为了展 开检测, 需要较长时间的事前准备, 并且价格昂贵, 许多患者 往往在犹豫之中错失了最佳治疗时间，导致治疗效果受到影 响。而 CT 血管造影 (CTA) 属于微创检查, 通常会给患者带来 二次伤害, 且价格与 DSA 相比相对便宜。但其同样具有一定 不足, 如需要多次平扫和增强扫描, 会对患者身体带来较大辐 射伤寒, 且扫描时间较长, 若患者不配合, 在扫描过程中头部 动作较大, 将会对检测准确率产生较大影响 ${ }^{[3]}$ 。根据以往的调 查研究可知, 自发性颉内出血患者在进行 CT 血管造影 (CTA) 时, 受到病情的影响, 难免会控制不住的小幅移动, 并导致扫 描结果受到影响,这也是 CT 血管造影( CTA)扫描准确率较低 的主要原因。

通过本次对常规血管造影术和双能量 CT 血管造影术检 测时长和准确率的对比分析, 可以发现应用双能量 CT 血管造 影术的观察组患者平均辐射剂量几乎比对照组患者小一倍左 右, 且观察组患者的平均扫描时间也远远短于对照组, 充分体 现出了双能量 CT 血管造影术在临床检验中的重要效果。同 时, 还可以看出观察组和对照组检测准确率相近, 且不具备统 计学意义, 因此并没有明显差异。另外, 双能量 CT 血管造影术 应用的优势主要体现在能够在同一时间进行平扫和增强扫 描,有效缩短了扫描时间,显著降低了患者的辐射剂量, 且优 化了操作流程, 提升了临床检验效率, 为推动医学进步、保护 人民身体健康奠定了良好的基础。

\section{5 结语}

综上所述,在自发性颅内出血疾病中应用双能量 CT 血管 造影术, 能够充分发挥出其扫描时间短、副作用小、准确性高 的优势, 对于医师及时准确地进行临床诊断、降低患者的致残 致死率具有重要意义, 可有效提升患者治疗满意度, 因此在临 床值得推广和加大应用。

\section{参考文献}

[1]杨帆,曾懿.双能量 CT 虚拟单能谱成像技术在 TIPS 术后支架 评估中的应用[J].中国中西医结合影像学杂志,2019,17(3):266-268.

[2]白爱国,马光慧, 滑炎卿.双能量 CT 血管造影的应用进展 [J].中 华临床医师杂志,2013,7(22):10325-10328

[3]费军,张朝利,刘军华,等.双能量 CT 血管造影在自发性颅内出 血中的沴断价值[J].中华老年心脑血管病杂志,2012,14(10):1039-1040. 